\title{
On the Delone property of $(-\beta)$-integers
}

\author{
Wolfgang Steiner \\ LIAFA, CNRS, Université Paris Diderot - Paris 7, \\ Case 7014, 75205 Paris Cedex 13, France \\ steiner@liafa.jussieu.fr
}

The $(-\beta)$-integers are natural generalisations of the $\beta$-integers, and thus of the integers, for negative real bases. They can be described by infinite words which are fixed points of anti-morphisms. We show that they are not necessarily uniformly discrete and relatively dense in the real numbers.

\section{Introduction}

We study the set of $(-\beta)$-integers for a real number $\beta>1$. This set is defined by

$$
\mathbb{Z}_{-\beta}=\bigcup_{n \geq 0}(-\beta)^{n} T_{-\beta}^{-n}(0),
$$

where $T_{-\beta}$ is the $(-\beta)$-transformation, defined by Ito and Sadahiro [5] as

$$
T_{-\beta}:\left[\frac{-\beta}{\beta+1}, \frac{1}{\beta+1}\right), \quad x \mapsto-\beta x-\left\lfloor\frac{\beta}{\beta+1}-\beta x\right\rfloor .
$$

Equivalently, a $(-\beta)$-integer is a real number of the form

$$
\sum_{k=0}^{n-1} d_{k}(-\beta)^{k}, \quad \text { with } \quad \frac{-\beta}{\beta+1} \leq \sum_{k=0}^{m-1} d_{k}(-\beta)^{k-m}<\frac{1}{\beta+1} \quad \text { for all } 1 \leq m \leq n,
$$

where $d_{0}, d_{1}, \ldots, d_{k-1}$ are integers. Examples of $(-\beta)$-transformations are depicted in Figure 1

Recall that the set of $\beta$-integers is defined by

$$
\mathbb{Z}_{\beta}=\mathbb{Z}_{\beta}^{+} \cup\left(-\mathbb{Z}_{\beta}^{+}\right) \quad \text { with } \quad \mathbb{Z}_{\beta}^{+}=\bigcup_{n \geq 0} \beta^{n} T_{\beta}^{-n}(0),
$$

where $T_{\beta}$ is the $\beta$-transformation,

$$
T_{\beta}:[0,1) \rightarrow[0,1), \quad x \mapsto \beta x-\lfloor\beta x\rfloor .
$$

These sets were introduced in the domain of quasicrystallography, see e.g. [2].

It is not difficult to see that $\mathbb{Z}_{-\beta}=\mathbb{Z}$ when $\beta \in \mathbb{Z}$, and that $\mathbb{Z}_{-\beta}=\{0\}$ when $\beta<\frac{1+\sqrt{5}}{2}$. For $\beta \geq \frac{1+\sqrt{5}}{2}$, Ambrož et al. [1] showed that $\mathbb{Z}_{-\beta}$ can be described by the fixed point of an anti-morphism on a possibly infinite alphabet. They also calculated explicitely the set of distances between consecutive $(-\beta)$-integers when $T_{-\beta}^{n}\left(\frac{-\beta}{\beta+1}\right) \leq 0$ and $T_{-\beta}^{2 n-1}\left(\frac{-\beta}{\beta+1}\right) \geq \frac{1-\lfloor\beta\rfloor}{\beta}$ for all $n \geq 1$. It seems to be difficult to extend their methods to the general case. For the case when $\beta$ is an Yrrap number, i.e., when $\left\{T_{-\beta}^{n}\left(\frac{-\beta}{\beta+1}\right) \mid n \geq 0\right\}$ is a finite set, a different approach can be found in [8]. The approach in Section 2 resembles that in [8], but it is simpler and works for general $\beta$. In Section 3 , we discuss the Delone property for sets $\mathbb{Z}_{-\beta}$. 


\section{Fixed point of an anti-morphism}

By Lemma 1 we can consider the set of $(-\beta)$-integers, $\beta>1$, as a special instance of the preimage of a point in $\left[\frac{-\beta}{\beta+1}, \frac{1}{\beta+1}\right)$ of the map

$$
\imath_{\beta}: \mathbb{R} \rightarrow\left[\frac{-\beta}{\beta+1}, \frac{1}{\beta+1}\right), \quad x \mapsto T_{-\beta}^{n}\left((-\beta)^{-n} x\right), \text { with } n \geq 0 \text { such that }(-\beta)^{-n} x \in\left(\frac{-\beta}{\beta+1}, \frac{1}{\beta+1}\right) .
$$

Since $T_{-\beta}\left((-\beta)^{-1} x\right)=x$ for all $x \in\left(\frac{-\beta}{\beta+1}, \frac{1}{\beta+1}\right)$, the map $\imath_{\beta}$ is well defined.

Lemma 1. For any $\beta>1$, we have

$$
\mathbb{Z}_{-\beta}=i_{\beta}^{-1}(0)
$$

Proof. If $x \in l_{\beta}^{-1}(0)$, then $T_{-\beta}^{n}\left((-\beta)^{-n} x\right)=0$ for some $n \geq 0$, thus $x \in(-\beta)^{n} T_{-\beta}^{-n}(0)$, i.e., $x \in \mathbb{Z}_{-\beta}$. On the other hand, $x \in \mathbb{Z}_{-\beta}$ implies that $x \in(-\beta)^{n} T_{-\beta}^{-n}(0)$ for some $n \geq 0$. If $x(-\beta)^{-n} \in\left(\frac{-\beta}{\beta+1}, \frac{1}{\beta+1}\right)$, this immediately implies that $\imath_{\beta}(x)=0$. If $(-\beta)^{-n} x=\frac{-\beta}{\beta+1}$, then

$$
T_{-\beta}^{n+2}\left((-\beta)^{-(n+2)} x\right)=T_{-\beta}^{n+2}\left(\frac{-1 / \beta}{\beta+1}\right)=T_{-\beta}^{n+1}\left(\frac{-\beta}{\beta+1}\right)=T_{-\beta}^{n+1}\left((-\beta)^{-n} x\right)=T_{-\beta}(0)=0,
$$

thus $\imath_{\beta}(x)=0$ as well.

Note that $\boldsymbol{l}_{\beta}(x)=x$ for all $x \in\left(\frac{-\beta}{\beta+1}, \frac{1}{\beta+1}\right)$. For other $x$, we use the following relation.

Lemma 2. For any $\beta>1, x \in \mathbb{R}$, we have

$$
\iota_{\beta}(-\beta x)=T_{-\beta}\left(\iota_{\beta}(x)\right) .
$$

Proof. Let $x \in \mathbb{R}$ with $(-\beta)^{-n} x \in\left(\frac{-\beta}{\beta+1}, \frac{1}{\beta+1}\right), n \geq 0$. Then we have

$$
\imath_{\beta}(-\beta x)=T_{-\beta}^{n+1}\left((-\beta)^{-(n+1)}(-\beta x)\right)=T_{-\beta} T_{-\beta}^{n}\left((-\beta)^{-n} x\right)=T_{-\beta}\left(\imath_{\beta}(x)\right) .
$$

An important role in the study of the $(-\beta)$-transformation is played by the orbit of the left endpoint $\frac{-\beta}{\beta+1}$. In the following, fix $\beta>1$, and let

$$
t_{n}=T_{-\beta}^{n}\left(\frac{-\beta}{\beta+1}\right) \quad(n \geq 0), \quad a_{n}=\left\lfloor-t_{0}-\beta t_{n-1}\right\rfloor \quad(n \geq 1) .
$$

(As usual, $\lfloor x\rfloor$ denotes the largest integer $\leq x$, and $\lceil x\rceil$ denotes the smallest integer $\geq x$.) Then $a_{1} a_{2} \cdots$ is the $(-\beta)$-expansion of $\frac{-\beta}{\beta+1}$, i.e.,

$$
t_{n}=\sum_{k=1}^{\infty} \frac{a_{n+k}}{(-\beta)^{k}} \quad \text { for all } n \geq 0
$$

see [5]. Setting $t_{-1}=\frac{1}{\beta+1}, t_{\infty}=0, \mathbb{N}_{\infty}=\{0,1,2, \ldots\} \cup\{\infty\}$, we consider open intervals

$$
J_{(i, j)}=\left(t_{2 i}, t_{2 j-1}\right) \quad \text { with } i, j \in \mathbb{N}_{\infty}, 0 \leq t_{2 i}<t_{2 j-1} \text { or } t_{2 i}<t_{2 j-1} \leq 0
$$

(where $2 \infty=\infty$ and $\infty-1=\infty$ ). We also set $a_{0}=a_{\infty}=0$, and

$$
\mathscr{A}=\left\{(i, j) \mid i, j \in \mathbb{N}_{\infty}, 0 \leq t_{2 i}<t_{2 j-1} \text { or } t_{2 i}<t_{2 j-1} \leq 0\right\} .
$$


(Here, $(i, j)$ is a pair of elements in $\mathbb{N}_{\infty}$, and not an open interval.) Let

$$
L_{\beta}((i, j))=t_{2 j-1}-t_{2 i} \quad((i, j) \in \mathscr{A})
$$

be the length of the interval $J_{(i, j)}$, and set

$$
L_{\beta}\left(v_{1} \cdots v_{k}\right)=L_{\beta}\left(v_{1}\right)+\cdots+L_{\beta}\left(v_{k}\right), \quad\left|v_{1} \cdots v_{k}\right|=k,
$$

for any word $v_{1} \cdots v_{k} \in \mathscr{A}^{*}$, where $\mathscr{A}^{*}$ denotes the free monoid over $\mathscr{A}$.

Let $\psi_{\beta}: \mathscr{A}^{*} \rightarrow \mathscr{A}^{*}$ be an anti-morphism, which is defined on $(i, j) \in \mathscr{A}$ by

$$
\psi_{\beta}((i, j))=(j, i+1) \quad \text { if } a_{2 i+1}=a_{2 j}, t_{2 i+1} t_{2 j} \geq 0,
$$

and otherwise by

$$
\psi_{\beta}((i, j))=\left\{\begin{array}{cl}
(j, \infty)((\infty, 0)(0, \infty))^{a_{2 i+1}-a_{2 j}}(\infty, i+1) & \text { if } t_{2 i+1}>0, t_{2 j}<0, \\
(j, 0)((0, \infty)(\infty, 0))^{a_{2 i+1}-a_{2 j}-1}(0, \infty)(\infty, i+1) & \text { if } t_{2 i+1}>0, t_{2 j} \geq 0, \\
(j, 0)((0, \infty)(\infty, 0))^{a_{2 i+1}-a_{2 j}-1}(0, i+1) & \text { if } t_{0}<t_{2 i+1} \leq 0, t_{2 j} \geq 0, \\
(j, \infty)(\infty, 0)((0, \infty)(\infty, 0))^{a_{2 i+1}-a_{2 j}-1}(0, i+1) & \text { if } t_{0}<t_{2 i+1} \leq 0, t_{2 j}<0, \\
(j, 0)((0, \infty)(\infty, 0))^{a_{2 i+1}-a_{2 j}-1} & \text { if } t_{2 i+1}=t_{0}, t_{2 j} \geq 0, \\
(j, \infty)(\infty, 0)((0, \infty)(\infty, 0))^{a_{2 i+1}-a_{2 j}-1} & \text { if } t_{2 i+1}=t_{0}, t_{2 j}<0 .
\end{array}\right.
$$

Here, anti-morphism means that $\psi_{\beta}(v w)=\psi_{\beta}(w) \psi_{\beta}(v)$ for all $v, w \in \mathscr{A}^{*}$. The anti-morphism $\psi_{\beta}$ is naturally extended to infinite words over $\mathscr{A}$. (Right infinite words are mapped to left infinite words and vice versa.)

Lemma 3. Let $\beta>1$. For any $u \in \mathscr{A}$, we have

$$
L_{\beta}\left(\psi_{\beta}(u)\right)=\beta L_{\beta}(u) .
$$

Moreover, for any $1 \leq \ell \leq\left|\psi_{\beta}(u)\right|, 0<x<L_{\beta}\left(v_{\ell}\right)$, with $\psi_{\beta}(u)=v_{1} \cdots v_{\left|\psi_{\beta}(u)\right|}$, we have

$$
T_{-\beta}\left(t_{2 j-1}-\beta^{-1} L_{\beta}\left(v_{1} \cdots v_{\ell-1}\right)-\beta^{-1} x\right)=t_{2 i^{\prime}}+x, \quad \text { where } u=(i, j), v_{\ell}=\left(i^{\prime}, j^{\prime}\right) .
$$

Proof. This follows from the definitions of $T_{-\beta}$ and $\psi_{\beta}$.

Let $\cdots u_{-1} u_{0} u_{1} \cdots \in \mathscr{A}^{\mathbb{Z}}$ be the fixed point of $\psi_{\beta}$ such that $u_{0}=(\infty, 0), u_{0} u_{1} \cdots$ is a fixed point of $\psi_{\beta}^{2}$ and $\cdots u_{-2} u_{-1}=\psi_{\beta}\left(u_{0} u_{1} \cdots\right)$, in particular $u_{-1}=(0, \infty)$. Let

$$
Y_{\beta}=\left\{y_{k} \mid k \in \mathbb{Z}\right\} \quad \text { with } \quad y_{k}=\left\{\begin{array}{cc}
L_{\beta}\left(u_{0} \cdots u_{k-1}\right) & \text { if } k \geq 0, \\
-L_{\beta}\left(u_{k} \cdots u_{-1}\right) & \text { if } k<0 .
\end{array}\right.
$$

Proposition 1. Let $\beta>1$. On every interval $\left(y_{k}, y_{k+1}\right), k \in \mathbb{Z}$, the map $\imath_{\beta}$ is a translation, with

$$
\iota_{\beta}\left(\left(y_{k}, y_{k+1}\right)\right)=J_{u_{k}}
$$


Proof. We have $l_{\beta}(x)=x$ on the intervals $\left(y_{0}, y_{1}\right)=\left(t_{\infty}, t_{-1}\right)=\left(0, \frac{1}{\beta+1}\right)$ and $\left(y_{-1}, y_{0}\right)=\left(t_{0}, t_{\infty}\right)=$ $\left(\frac{-\beta}{\beta+1}, 0\right)$, thus the statement of the proposition holds for $\left(y_{k}, y_{k+1}\right) \subset\left(\frac{-\beta}{\beta+1}, \frac{1}{\beta+1}\right)$.

Assume that the statement holds for $k \in \mathbb{Z}$. By Lemma 3, we have $(-\beta)\left(y_{k}, y_{k+1}\right)=\left(y_{k^{\prime}}, y_{k^{\prime}+\left|\psi_{\beta}\left(u_{k}\right)\right|}\right)$ and $\psi_{\beta}\left(u_{k}\right)=u_{k^{\prime}} \cdots u_{k^{\prime}+\left|\psi_{\beta}\left(u_{k}\right)\right|-1}$, with $k^{\prime}=-\left|\psi_{\beta}\left(u_{0} \cdots u_{k}\right)\right|$ if $k \geq 0, k^{\prime}=\left|\psi_{\beta}\left(u_{k+1} \cdots u_{-1}\right)\right|$ if $k<0$. Then Lemma2, the assumption $v_{\beta}\left(\left(y_{k}, y_{k+1}\right)\right)=J_{u_{k}}$, and Lemma 3 yield that

$$
\begin{aligned}
\iota_{\beta}\left(\left(y_{k^{\prime}+\ell}, y_{k^{\prime}+\ell+1}\right)\right) & =T_{-\beta}\left(\imath_{\beta}\left((-\beta)^{-1}\left(y_{k^{\prime}+\ell}, y_{k^{\prime}+\ell+1}\right)\right)\right) \\
& =T_{-\beta}\left(\iota_{\beta}\left(\left(y_{k+1}-\beta^{-1} L_{\beta}\left(u_{k^{\prime}} \cdots u_{k^{\prime}+\ell}\right), y_{k+1}-\beta^{-1} L_{\beta}\left(u_{k^{\prime}} \cdots u_{k^{\prime}+\ell-1}\right)\right)\right)\right) \\
& =T_{-\beta}\left(\left(t_{2 j-1}-\beta^{-1} L_{\beta}\left(u_{k^{\prime}} \cdots u_{k^{\prime}+\ell}\right), t_{2 j-1}-\beta^{-1} L_{\beta}\left(u_{k^{\prime}} \cdots u_{k^{\prime}+\ell-1}\right)\right)\right) \\
& =J_{u_{k^{\prime}+\ell}}
\end{aligned}
$$

for all $0 \leq \ell<\left|\psi_{\beta}\left(u_{k}\right)\right|$, where $u_{k}=(i, j)$.

By induction on $n$, we obtain for every $n \geq 0$ that the statement of the proposition holds for all $k \in \mathbb{Z}$ with $\left(y_{k}, y_{k+1}\right) \subset(-\beta)^{n}\left(\frac{-\beta}{\beta+1}, \frac{1}{\beta+1}\right)$, thus it holds for all $k \in \mathbb{Z}$.

Now we describe the set $Y_{\beta}=\left\{y_{k} \mid k \in \mathbb{Z}\right\}$, which is left out by the intervals $\left(y_{k}, y_{k+1}\right)$.

Lemma 4. For any $\beta>1$, we have

$$
Y_{\beta}=\mathbb{Z}_{-\beta} \cup \bigcup_{m, n \geq 0}(-\beta)^{m+n} T_{-\beta}^{-n}\left(\frac{-\beta}{\beta+1}\right) .
$$

Proof. First note that

$$
\bigcup_{m, n \geq 0}(-\beta)^{m+n} T_{-\beta}^{-n}\left(\frac{-\beta}{\beta+1}\right)=\bigcup_{m \geq 0}(-\beta)^{m} i_{\beta}^{-1}\left(\frac{-\beta}{\beta+1}\right),
$$

similarly to Lemma11. Indeed, $x \in t_{\beta}^{-1}\left(t_{0}\right)$ is equivalent to $(-\beta)^{-n} x \in T_{-\beta}^{-n}\left(t_{0}\right) \cap\left(t_{0}, t_{-1}\right)$ for some $n \geq 0$. In the remaining case $(-\beta)^{-n} x=t_{0} \in T_{-\beta}^{-n}\left(t_{0}\right)$, we have $x \in(-\beta) l_{\beta}^{-1}\left(t_{0}\right)$ since $T_{-\beta}^{n+1}\left((-\beta)^{-(n+2)} x\right)=$ $T_{-\beta}^{n}\left(t_{0}\right)=t_{0}$. Note also that $(-\beta)^{m} i_{\beta}^{-1}\left(t_{0}\right) \subseteq i_{\beta}^{-1}\left(t_{m}\right)$.

Since $t_{0} \notin J_{u_{k}}$ and $0 \notin J_{u_{k}}$ for all $k \in \mathbb{Z}$ by the definition of $\mathscr{A}$, Proposition 1 implies that $\imath_{\beta}^{-1}(0) \cup$ $l_{\beta}^{-1}\left(t_{0}\right) \subseteq Y_{\beta}$, thus $\mathbb{Z}_{-\beta} \subseteq Y_{\beta}$ by Lemma1 Since $(-\beta) Y_{\beta} \subseteq Y_{\beta}$ by Lemma3, we obtain $(-\beta)^{m} l_{\beta}^{-1}\left(t_{0}\right) \subseteq$ $Y_{\beta}$ for every $m \geq 0$ as well.

Let now $x \in Y_{\beta} \backslash\{0\}$. Then there exists some $m \geq 0$ such that $(-\beta)^{-m} x \in Y_{\beta}$ and $(-\beta)^{-m-1} x \notin Y_{\beta}$, i.e., $(-\beta)^{-m-1} x \in\left(y_{k}, y_{k+1}\right)$ for some $k \in \mathbb{Z}$. By Lemma 2 and the definition of $\psi_{\beta}$, we obtain that $\boldsymbol{l}_{\beta}\left((-\beta)^{-m} x\right) \in\left\{t_{0}, 0\right\}$, i.e., $x \in(-\beta)^{m} \boldsymbol{l}_{\beta}^{-1}\left(t_{0}\right)$ or $x \in(-\beta)^{m} \mathbb{Z}_{-\beta} \subseteq \mathbb{Z}_{-\beta}$. Since $0 \in \mathbb{Z}_{-\beta}$, this proves the lemma.

Theorem 1. Let $\beta>1, x \in \mathbb{R}$. Then $x \in \mathbb{Z}_{-\beta}$ if and only if $x=y_{k}$ for some $k \in \mathbb{Z}$ with $t_{2 j-1}=0$ or $t_{2 i}=0$, where $u_{k-1}=\left(i^{\prime}, j\right), u_{k}=\left(i, j^{\prime}\right)$.

Proof. Since $\mathbb{Z}_{-\beta} \subseteq Y_{\beta}$ by Lemma 4 it is sufficient to consider $x=y_{k}, k \in \mathbb{Z}$. As in the proof of Lemma4] let $m \geq 0$ be such that $(-\beta)^{-m} x \in Y_{\beta}$ and $(-\beta)^{-m-1} x \notin Y_{\beta}$. Then we have $\imath_{\beta}\left((-\beta)^{-m} x\right) \in\left\{t_{0}, 0\right\}$.

If $\iota_{\beta}\left((-\beta)^{-m} x\right)=0$, then $\imath_{\beta}(x)=T_{-\beta}^{m}(0)=0$. Moreover, $\iota_{\beta}$ is continuous at $(-\beta)^{-m} x$ in this case. Together with the continuity of $T_{-\beta}^{m}$ at 0 , this implies that $\iota_{\beta}$ is continuous at $x$, i.e., $u_{k-1}=\left(i^{\prime}, j\right)$ with $t_{2 j-1}=0$ and $u_{k}=\left(i, j^{\prime}\right)$ with $t_{2 i}=0$.

If $\imath_{\beta}\left((-\beta)^{-m} x\right)=t_{0}$, then $\imath_{\beta}$ is right continuous at $(-\beta)^{-m} x$, and its limit from the left is $t_{-1}$. We obtain that $u_{\beta}(x)=t_{m}, u_{k-1}=\left(i^{\prime},\lceil m / 2\rceil\right)$ and $u_{k}=\left(\lfloor m / 2\rfloor, j^{\prime}\right)$ for some $i^{\prime}, j^{\prime} \in \mathbb{N}_{\infty}$. Let $i=\lfloor m / 2\rfloor$, 
$j=\lceil m / 2\rceil$. Since $2 i=m$ if $m$ is even and $2 j-1=m$ if $m$ is odd, $x \in \mathbb{Z}_{-\beta}$ implies that $t_{2 i}=0$ or $t_{2 j-1}=0$. On the other hand, $t_{2 i}=0$ or $t_{2 j-1}=0$ yields that $t_{m-1}=0$ or $t_{m}=0$. Since $t_{m-1}=0$ implies $t_{m}=0$, we must have $x \in \mathbb{Z}_{-\beta}$.

By Theorem 1 the study of $\mathbb{Z}_{-\beta}$ is reduced to the study of the fixed point of $\psi_{\beta}$. Note that $(i, j)$ and $\left(i^{\prime}, j^{\prime}\right)$ can be identified when $t_{2 i}=t_{2 i^{\prime}}$ and $t_{2 j-1}=t_{2 j^{\prime}-1}$. After identification, $\mathscr{A}$ is finite if and only if $\left\{t_{n} \mid n \geq 0\right\}$ is a finite set, i.e., if $\beta$ is an Yrrap number.

With the help of $\psi_{\beta}$, we can construct an anti-morphism describing the structure of $\mathbb{Z}_{-\beta}$ (for $\beta \geq$ $\left.\frac{1+\sqrt{5}}{2}\right)$, similarly to [8]. First note that $\beta \geq \frac{1+\sqrt{5}}{2}$ implies $a_{1}=1, t_{1} \geq 0$, or $a_{1} \geq 2$, thus $\psi_{\beta}^{2}((\infty, 0))=$ $\psi_{\beta}((0, \infty))$ starts with $(\infty, 0)(0, \infty)$. Therefore, $L_{\beta}((\infty, 0)(0, \infty))=t_{-1}-t_{0}=1$ is the smallest positive element of $\mathbb{Z}_{-\beta}$. Using Theorem 1 and $(-\beta) \mathbb{Z}_{-\beta} \subseteq \mathbb{Z}_{-\beta}$, the word $\psi_{\beta}((\infty, 0)(0, \infty))$ determines the set $\mathbb{Z}_{-\beta} \cap[-\beta, 0]$. Splitting up $\psi_{\beta}((\infty, 0)(0, \infty))$ according to Theorem 1 and applying $\psi_{\beta}$ on each of the factors yields the set $\mathbb{Z}_{-\beta} \cap\left[0, \beta^{2}\right]$, etc. Consider all these factors, i.e., all words $u_{k} \cdots u_{k^{\prime}-1}$ between consecutive elements $y_{k}, y_{k^{\prime}} \in \mathbb{Z}_{-\beta}$, as letters. Using the described strategy, we define an anti-morphism $\varphi_{-\beta}$ on words consisting of these letters. Then the fixed point of $\varphi_{-\beta}$ codes the distances between the elements of $\mathbb{Z}_{-\beta}$, see the two simple examples below. For more complicated examples, we refer to [8]. By [1, 8], the alphabet is finite if and only if $\beta$ is an Yrrap number.
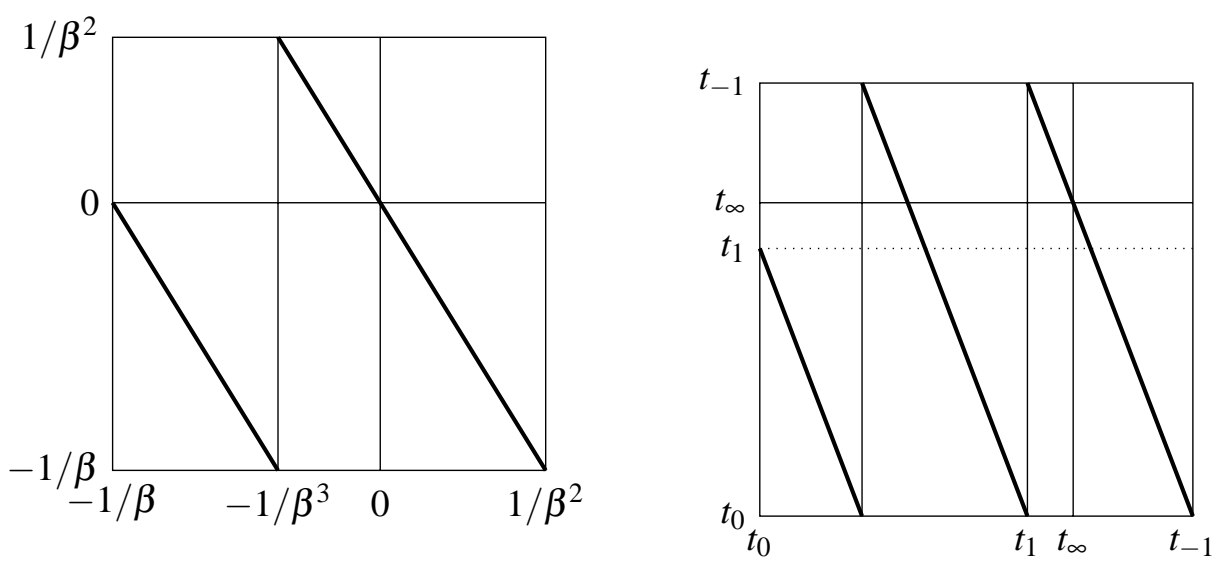

Figure 1: The $(-\beta)$-transformation for $\beta=\frac{1+\sqrt{5}}{2}$ (left) and $\beta=\frac{3+\sqrt{5}}{2}$ (right).

Example 1. Let $\beta=\frac{3+\sqrt{5}}{2} \approx 2.618$, i.e., $\beta^{2}=3 \beta-1$. Then we have $t_{1}=\frac{\beta^{2}}{\beta+1}-2=\frac{-1 / \beta}{\beta+1}$ and $t_{2}=$ $\frac{1}{\beta+1}-1=t_{0}$. Therefore, we can identify $(0, \infty)$ and $(1, \infty)$, and obtain

$$
\begin{aligned}
\psi_{\beta}: \quad(\infty, 0) & \mapsto(0, \infty), \\
(0, \infty) & \mapsto(\infty, 0)(0, \infty)(\infty, 0)(0,1), \\
(0,1) & \mapsto(0, \infty)(\infty, 0)(0,1) .
\end{aligned}
$$

The two-sided fixed point $\cdots u_{-2} u_{-1} u_{0} u_{1} \cdots$ of $\psi_{\beta}$ is equal to

$$
\cdots(0, \infty)(\infty, 0)(0,1)(0, \infty)(\infty, 0)(0, \infty)(\infty, 0)(0,1)(0, \infty)^{\cdot}(\infty, 0)(0, \infty)(\infty, 0)(0,1) \cdots .
$$

Applying $\psi_{\beta}$ to $(\infty, 0)(0, \infty)$ and to the factors described by Theorem 1 yields

$$
\begin{aligned}
(\infty, 0)(0, \infty) & \mapsto(\infty, 0)(0, \infty)(\infty, 0)(0,1)(0, \infty), \\
(\infty, 0)(0,1)(0, \infty) & \mapsto(\infty, 0)(0, \infty)(\infty, 0)(0,1)(0, \infty)(\infty, 0)(0,1)(0, \infty) .
\end{aligned}
$$


Therefore, setting $A=(\infty, 0)(0, \infty)$ and $B=(\infty, 0)(0,1)(0, \infty)$, the fixed point

\section{$\cdots A B B A B A B B A B B A B \cdot A B B A B A B B A B B A B \cdots$}

of the anti-morphism

$$
\varphi_{-\beta}: \quad A \mapsto A B, \quad B \mapsto A B B,
$$

describes the set of $(-\beta)$-integers, see Figure 2 The distances between consecutive elements of $\mathbb{Z}_{-\beta}$ are $L_{\beta}(A)=1$ and $L_{\beta}(B)=\beta-1>1$.

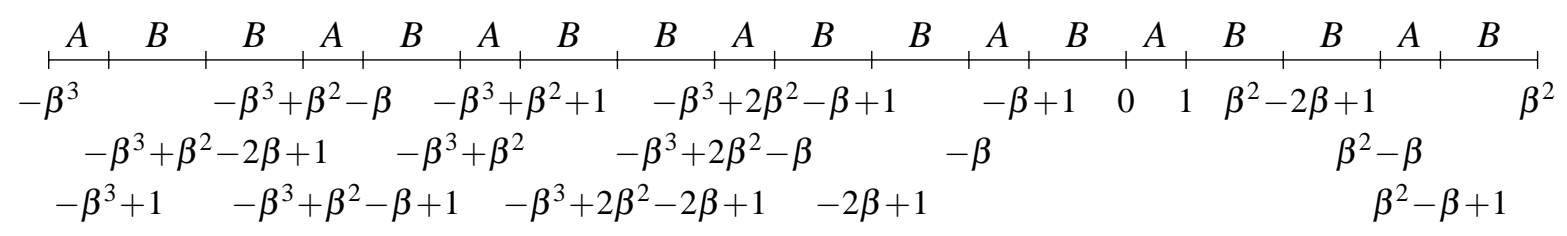

Figure 2: The set $\mathbb{Z}_{-\beta} \cap\left[-\beta^{3}, \beta^{2}\right], \beta=(3+\sqrt{5}) / 2$.

Example 2. Let $\beta=\frac{1+\sqrt{5}}{2} \approx 1.618$, i.e., $\beta^{2}=\beta+1$. Then we have $t_{1}=\frac{\beta^{2}}{\beta+1}-1=0=t_{\infty}$. Identifying $(0, \infty)$ and $(0,1)$, we obtain

$$
\psi_{\beta}: \quad(\infty, 0) \mapsto(0, \infty), \quad(0, \infty) \mapsto(\infty, 0)(0, \infty)
$$

The two-sided fixed point $\cdots u_{-2} u_{-1} \cdot u_{0} u_{1} \cdots$ of $\psi_{\beta}$ is equal to

$$
\cdots(\infty, 0)(0, \infty)(0, \infty)^{\cdot}(\infty, 0)(0, \infty)(\infty, 0)(0, \infty)(0, \infty) \cdots
$$

The words $u_{k} \cdots u_{k^{\prime}-1}$ between consecutive elements $y_{k}, y_{k^{\prime}} \in \mathbb{Z}_{-\beta}$ are $A=(\infty, 0)(0, \infty)$ and $B=(0, \infty)$, since

$$
(\infty, 0)(0, \infty) \mapsto(\infty, 0)(0, \infty)(0, \infty), \quad(0, \infty) \mapsto(\infty, 0)(0, \infty)
$$

Note that $B$ does not start with a letter $(i, j)$ with $t_{2 i}=0$, thus $\imath_{\beta}$ is discontinuous at the corresponding points $y_{k} \in \mathbb{Z}_{-\beta}$. The fixed point

$$
\cdots A A B A A B A B A A B A B \cdot A A B A A B A B A A B A A B A B A A B A B \cdots
$$

of the anti-morphism

$$
\varphi_{-\beta}: \quad A \mapsto A B, \quad B \mapsto A,
$$

describes the set of $(-\beta)$-integers, with $L_{\beta}(A)=1$ and $L_{\beta}(B)=\beta-1<1$, see Figure 3 Note that $(-\beta)^{n}$ can also be represented as $(-\beta)^{n+2}+(-\beta)^{n+1}$.

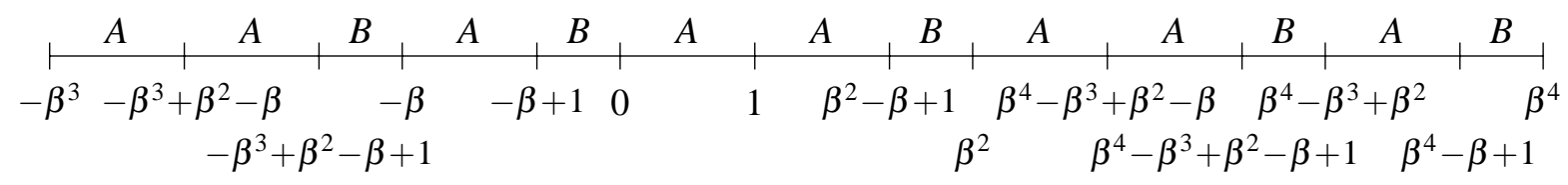

Figure 3: The $(-\beta)$-integers in $\left[-\beta^{3}, \beta^{4}\right], \beta=(1+\sqrt{5}) / 2$. 


\section{Delone property}

A set $S \subset \mathbb{R}$ is called Delone set (or Delaunay set) if it is uniformly discrete and relatively dense; i.e., if there are numbers $R>r>0$, such that each interval of length $r$ contains at most one point of $S$, and every interval of length $R$ contains at least one point of $S$.

If $\beta$ is an Yrrap number, then the set of distances between consecutive $(-\beta)$-integers is finite by [1, 8], thus $\mathbb{Z}_{-\beta}$ is a Delone set. For general $\beta$, we show in this section that $\mathbb{Z}_{-\beta}$ need neither be uniformly discrete nor relatively dense.

\subsection{Uniform discreteness}

Since every point $x \in Y_{\beta}$ is separated from $Y_{\beta} \backslash\{x\}$ by an interval around $x, Y_{\beta}$ is discrete, and the same holds for $\mathbb{Z}_{-\beta}$. It is well known that $\mathbb{Z}_{\beta}$ is uniformly discrete if and only if 0 is not an accumulation point of $\left\{T_{\beta}^{n}(1) \mid n \geq 0\right\}$ (where $T_{\beta}(1)=\beta-\lfloor\beta\rfloor$ ). For $(-\beta)$-integers, the situation is more complicated.

Proposition 2. Let $\beta>1$. If 0 is not an accumulation point of $\left\{T_{-\beta}^{2 n-1}\left(\frac{-\beta}{\beta+1}\right)>0 \mid n \geq 1\right\}$, then the set $\mathbb{Z}_{-\beta}$ is uniformly discrete.

Proof. When $\beta$ is an Yrrap number, then $\left\{L_{\beta}\left(u_{k}\right) \mid k \in \mathbb{Z}\right\}$ is finite, thus $\mathbb{Z}_{-\beta}$ is uniformly discrete. Therefore, assume that $\beta$ is not an Yrrap number, in particular that $t_{n} \neq 0$ for all (finite) $n \geq 0$, with the notation of Section 2 . Then $\imath_{\beta}$ is continuous at every point $y_{k} \in \mathbb{Z}_{-\beta}, k \in \mathbb{Z}$, thus $u_{k}=(i, j)$ with $t_{2 i}=0$. Since $L_{\beta}\left(u_{k}\right) \geq \inf \left\{t_{2 n-1}>0 \mid n \geq 0\right\}$, the set $\mathbb{Z}_{-\beta}$ is uniformly discrete if $\inf \left\{t_{2 n-1}>0 \mid n \geq 1\right\}>0$.

In order to give examples of $\beta$ where $\mathbb{Z}_{-\beta}$ is not uniformly discrete or not relatively dense, we need to know which sequences are possible $(-\beta)$-expansions of $\frac{-\beta}{\beta+1}$. The corresponding problem for $\beta$ expansions was solved by Parry [7]. Góra [4, Theorem 25] gave an answer to a more general question, but his theorem is incorrect, as noticed in [3]. However, Góra proved the following result, where $\leq_{\text {alt }}$ denotes the alternate order on words, i.e., $x_{1} x_{2} \cdots<_{\text {alt }} y_{1} y_{2} \cdots$ if $(-1)^{n}\left(x_{n+1}-y_{n+1}\right)<0, x_{1} \cdots x_{n}=y_{1} \cdots y_{n}$ for some $n \geq 0$.

Lemma 5. Let $a_{1} a_{2} \cdots$ be a sequence of non-negative integers satisfying $a_{n+1} a_{n+2} \cdots \leq_{\mathrm{alt}} a_{1} a_{2} \cdots$ for all $n \geq 1$, with $a_{1} \geq 2$. Then there exists a unique $a_{1} \leq \beta \leq a_{1}+1$ such that

$$
\sum_{k=1}^{\infty} \frac{a_{k}}{(-\beta)^{k}}=\frac{-\beta}{\beta+1}, \quad \text { and } \quad \frac{-\beta}{\beta+1} \leq \sum_{k=1}^{\infty} \frac{a_{n+k}}{(-\beta)^{k}} \leq \frac{1}{\beta+1} \quad \text { for all } n \geq 1 .
$$

Proof. As the generalised $\beta$-transformations in [4] with $E=(1, \ldots, 1)$ are intimately related to our $T_{-\beta}$ (see the introduction of [6]), the lemma follows from the proof of Theorem 25 in [4].

The flaw in [4, Theorem 25] is that $\sum_{k=1}^{\infty} \frac{a_{n+k}}{(-\beta)^{k}}=\frac{1}{\beta+1}$ cannot be excluded, which would be necessary for $a_{1} a_{2} \cdots$ to be the $(-\beta)$-expansion of $\frac{-\beta}{\beta+1}$. Indeed, the sequence $a_{1} a_{2} \cdots=2(10)^{\omega}$ satisfies the conditions of Lemma 5 and yields $\beta=2$, but $\sum_{k=1}^{\infty} \frac{a_{k+2}}{(-2)^{k}}=\frac{1}{3}$; the $(-2)$-expansion of $\frac{-2}{3}$ is $2^{\omega}$. In order to avoid this problem, we define a relation $<_{\text {alt }}^{\prime}$ by $x_{1} x_{2} \cdots<_{\text {alt }}^{\prime} y_{1} y_{2} \cdots$ if $(-1)^{n}\left(x_{n+1}-y_{n+1}\right)<-1$, $x_{1} \cdots x_{n}=y_{1} \cdots y_{n}$ for some $n \geq 0$.

Lemma 6. Let $a_{1} a_{2} \cdots$ and $\beta$ be as in Lemma 5 If $a_{n+1} a_{n+2} \cdots<_{\text {alt }}^{\prime} a_{1} a_{2} \cdots$ for all $n \geq 2$ such that $a_{n}=0$, then $a_{1} a_{2} \cdots$ is the $(-\beta)$-expansion of $\frac{-\beta}{\beta+1}$. 
Proof. We have to show that $\sum_{k=1}^{\infty} \frac{a_{n-1+k}}{(-\beta)^{k}}<\frac{1}{\beta+1}$ for all $n \geq 2$. If $a_{n}>0$, then this inequality follows from $\sum_{k=1}^{\infty} \frac{a_{n-1+k}}{(-\beta)^{k}} \leq \frac{-1}{\beta}+\frac{1}{\beta+1}<0$. If $a_{n}=0$, then we have some $m \geq 0$ such that $(-1)^{m}\left(a_{n+m+1}-a_{m+1}\right) \leq-2$, $a_{n+1} \cdots a_{n+m}=a_{1} \cdots a_{m}$. This implies that

$$
\begin{aligned}
& \sum_{k=1}^{\infty} \frac{a_{n+k}}{(-\beta)^{k}}=\sum_{k=1}^{m} \frac{a_{k}}{(-\beta)^{k}}+\frac{1}{(-\beta)^{m}} \sum_{k=1}^{\infty} \frac{a_{n+m+k}}{(-\beta)^{k}} \\
& \geq \sum_{k=1}^{m} \frac{a_{k}}{(-\beta)^{k}}+\frac{a_{m+1}}{(-\beta)^{m+1}}+\frac{2-\frac{\beta}{\beta+1}}{\beta^{m+1}}>\sum_{k=1}^{m+1} \frac{a_{k}}{(-\beta)^{k}}+\frac{\frac{\beta}{\beta+1}}{\beta^{m+1}} \geq \sum_{k=1}^{\infty} \frac{a_{k}}{(-\beta)^{k}}=\frac{-\beta}{\beta+1},
\end{aligned}
$$

thus $\sum_{k=1}^{\infty} \frac{a_{n-1+k}}{(-\beta)^{k}}=\frac{1}{-\beta} \sum_{k=1}^{\infty} \frac{a_{n+k}}{(-\beta)^{k}}<\frac{1}{\beta+1}$.

Proposition 3. Let $a_{1} a_{2} \cdots=3010^{3} 10^{5} 1 \cdots$. Then $a_{1} a_{2} \cdots$ is the $(-\beta)$-expansion of $\frac{-\beta}{\beta+1}$ for some $\beta>1$, and $\mathbb{Z}_{-\beta}$ is not uniformly discrete.

Proof. By Lemmas 5 and 6, there exists a $\beta>1$ such that $a_{1} a_{2} \cdots$ is the $(-\beta)$-expansion of $\frac{-\beta}{\beta+1}$. Since $a_{2 n} a_{2 n+1} \cdots$ starts with an odd number of zeros for all $n \geq 1$, we have $t_{2 n-1}>0$ for all $n \geq 0$. Therefore, induction on $n$ yields that $\psi_{\beta}^{2 n}((\infty, 0))$ ends with $(\infty, n)$, and $\psi_{\beta}^{2 n+1}((\infty, 0))$ starts with $(n, \infty)$ for all $n \geq 0$. This implies that $\psi_{\beta}^{2 n+1}((\infty, 0)(0, \infty))$ contains the factor $(\infty, n)(n, \infty)$ for all $n \geq 0$, and $L_{\beta}((\infty, n)(n, \infty))=t_{2 n-1}-t_{2 n}$ is a distance between consecutive $(-\beta)$-integers. For any $k \geq 1$, we have $a_{k(k-1)+2} \cdots a_{k(k+1)+1}=0^{2 k-1} 1$, thus $0<t_{k(k-1)+1}<\beta^{-2 k} \frac{\beta^{2}}{\beta+1}$ and $-\beta^{-2 k} \frac{\beta^{3}}{\beta+1}<t_{k(k-1)+2}<0$. Therefore, the distance between consecutive elements of $\mathbb{Z}_{-\beta}$ can be arbitrarily small.

The following proposition shows that the converse of Proposition 2 is not true.

Proposition 4. Let $a_{1} a_{2} \cdots=300320000300322000000003003200003003222 \cdots$ be a fixed point of the morphism

$$
\sigma_{1}: 3 \mapsto 30032,2 \mapsto 2,0 \mapsto 00 .
$$

Then $a_{1} a_{2} \cdots$ is the $(-\beta)$-expansion of $\frac{-\beta}{\beta+1}$ for $a \beta>1$, inf $\left\{T_{-\beta}^{2 n-1}\left(\frac{-\beta}{\beta+1}\right)>0 \mid n \geq 1\right\}=0$ and $\mathbb{Z}_{-\beta}$ is uniformly discrete.

Proof. When $a_{n+1}=3, n \geq 1$, then $a_{n+1} a_{n+2} \cdots$ starts with $\sigma_{1}^{k}(3) 2$ for some $k \geq 0$. Since $\left|\sigma_{1}^{k}(3)\right|$ is odd for all $k \geq 0$, we have $\sigma_{1}^{k}(3) 2<_{\text {alt }}^{\prime} \sigma_{1}^{k}(3) 0$, thus $a_{n+1} a_{n+2} \cdots<_{\text {alt }}^{\prime} a_{1} a_{2} \cdots$. Since $a_{n}=0$ implies $a_{n+1} \in\{0,3\}$, the conditions of Lemmas 5 and 6 are satisfied, i.e., there exists a $\beta>1$ such that $a_{1} a_{2} \ldots$ is the $(-\beta)$-expansion of $\frac{-\beta}{\beta+1}$.

Let $n_{k}=\left|\sigma_{1}^{k}(300)\right|+\left|\sigma_{1}^{k-1}(3)\right|+1, k \geq 1$. Then $a_{n_{k}+1} \cdots a_{n_{k}+2^{k}}=0^{2^{k}-1} 3$, thus we have $t_{n_{k}}>0$ and $\lim _{k \rightarrow \infty} t_{n_{k}}=0$. Since $n_{k}$ is odd, this yields that $\inf \left\{t_{2 n-1}>0 \mid n \geq 1\right\}=0$.

Let $n \geq 1$ with $t_{2 n-1}>0$. Then $a_{2 n} a_{2 n+1} \cdots$ starts with an odd number of zeros, thus $a_{1} \cdots a_{2 n-1}$ ends with $\sigma_{1}^{k}(3) 0^{2 j-1}$ for some $k \geq 0,1 \leq j \leq 2^{k-1}$. Let $2 m=\left|\sigma_{1}^{k}(3)\right|+2 j-1$. Recall that $\cdots u_{-1} u_{0} u_{1} \cdots$ is a fixed point of $\psi_{\beta}$. Any letter $u_{k}=(i, n), i \in \mathbb{N}_{\infty}$, occurs only in $\psi_{\beta}^{2 m}\left(\left(i^{\prime}, n-m\right)\right), i^{\prime} \in \mathbb{N}_{\infty}$ with $t_{0} \leq t_{2 i^{\prime}}<t_{2 n-2 m-1}$. Since $a_{1} \cdots a_{2 m}=a_{2 n-2 m} \cdots a_{2 n-1}$, we obtain that $t_{2 m} \leq t_{2 i}<t_{2 n-1}$. Moreover, $a_{2 m+1} a_{2 m+2} \cdots$ starts with $0^{2^{k}-2 j+1} 3$, thus $t_{2 m}>0$. Now, the continuity of $\imath_{\beta}$ at every point in $\mathbb{Z}_{-\beta}$ yields that $y_{k} \notin \mathbb{Z}_{-\beta}$ if $u_{k}=(i, n), n \geq 1$. Therefore, $y_{k} \in \mathbb{Z}_{-\beta}$ implies that $u_{k}=(\infty, 0)$, hence $\mathbb{Z}_{-\beta}$ is uniformly discrete. 


\subsection{Relative denseness}

Since the distance between consecutive $\beta$-integers is at most $1, \mathbb{Z}_{\beta}$ is always relatively dense. We show that this is not always true for $\mathbb{Z}_{-\beta}$.

Proposition 5. Let $a_{1} a_{2} \cdots=31232123123221231232123123222 \cdots$ be a fixed point of the morphism

$$
\sigma_{2}: 3 \mapsto 31232,2 \mapsto 2,1 \mapsto 1
$$

Then $a_{1} a_{2} \cdots$ is the $(-\beta)$-expansion of $\frac{-\beta}{\beta+1}$ for $a \beta>1$, and $\mathbb{Z}_{-\beta}$ is not relatively dense.

Proof. Since $\left|\sigma_{2}^{k}(3)\right|$ is odd for all $k \geq 0$, we have $\sigma_{2}^{k}(3) 2<_{\text {alt }} \sigma_{2}^{k}(3) 1$, thus $a_{1} a_{2} \cdots$ satisfies the conditions of Lemma 5 . Since $a_{n}>0$ for all $n \geq 2$, the condition of Lemma 6 holds trivially. Therefore, there exists a $\beta>1$ such that $a_{1} a_{2} \cdots$ is the $(-\beta)$-expansion of $\frac{-\beta}{\beta+1}$.

Next we show that, for any $k \geq 0, \psi_{\beta}^{\left|\sigma_{2}^{k}(3)\right|}((\infty, 0))$ starts with

$$
\left(\left\lfloor\left|\sigma_{2}^{k}(3)\right| / 2\right\rfloor,\left\lceil\left|\sigma_{2}^{k-1}(3)\right| / 2\right\rceil\right) \cdots\left(\left\lfloor\left|\sigma_{2}^{1}(3)\right| / 2\right\rfloor,\left\lceil\left|\sigma_{2}^{0}(3)\right| / 2\right\rceil\right)(0, \infty) .
$$

We have $\psi_{\beta}((\infty, 0))=(0, \infty)$ and

$$
\psi_{\beta}^{4}((0, \infty))=\cdots \psi_{\beta}^{3}((0,1))=\psi_{\beta}^{2}((1, \infty)) \cdots=\cdots \psi_{\beta}((\infty, 0)(0,2))=(2,1)(0, \infty) \cdots,
$$

where we have used that $a_{1}>a_{2}, a_{4}=a_{1}$, and $a_{n}>0$ for all $n \geq 1$, which implies $t_{n}<0$ for all $n \geq 1$. This yields the statement for $k=0$ and $k=1$. Supose that $\psi_{\beta}^{\left|\sigma_{2}^{k}(3)\right|}((\infty, 0))$ starts with (1) for some $k \geq 1$. Then we have

$$
\begin{aligned}
\psi_{\beta}^{\left|\sigma_{2}^{k+1}(3)\right|}((\infty, 0))= & \psi_{\beta}^{\left|\sigma_{2}^{k}(3)\right|+3}\left(\left(\left\lfloor\left|\sigma_{2}^{k}(3)\right| / 2\right\rfloor,\left\lceil\left|\sigma_{2}^{k-1}(3)\right| / 2\right\rceil\right)\right) \cdots \\
= & \cdots \psi_{\beta}^{\left|\sigma_{2}^{k}(3)\right|+2}\left(\left(0,\left\lceil\left|\sigma_{2}^{k}(3)\right| / 2\right\rceil\right)\right) \\
= & \psi_{\beta}^{\left|\sigma_{2}^{k}(3)\right|+1}\left(\left(\left\lceil\left|\sigma_{2}^{k}(3)\right| / 2\right\rceil, \infty\right)\right) \cdots \\
= & \cdots \psi_{\beta}^{\left|\sigma_{2}^{k}(3)\right|}\left((\infty, 0)\left(0,\left\lceil\left|\sigma_{2}^{k}(3)\right| / 2\right\rceil+1\right)\right) \\
= & \left(2\left\lceil\left|\sigma_{2}^{k}(3)\right| / 2\right\rceil,\left\lceil\left|\sigma_{2}^{k}(3)\right| / 2\right\rceil\right) \\
& \left.\quad\left(\left\lfloor\left|\sigma_{2}^{k}(3)\right| / 2\right\rfloor,\left\lceil\left|\sigma_{2}^{k-1}(3)\right| / 2\right\rceil\right) \cdots\left(|| \sigma_{2}^{1}(3) \mid / 2\right\rfloor,\left\lceil\left|\sigma_{2}^{0}(3)\right| / 2\right\rceil\right)(0, \infty) \cdots,
\end{aligned}
$$

where we have used the relations $a_{\left|\sigma_{2}^{k}(3)\right|}=2>1=a_{\left|\sigma_{2}^{k-1}(3)\right|+1}, a_{1}>a_{\left|\sigma_{2}^{k}(3)\right|+1}$, and $a_{1} \cdots a_{\left|\sigma_{2}^{k}(3)\right|}=$ $a_{\left|\sigma_{2}^{k}(3)\right|+3} \cdots a_{2\left|\sigma_{2}^{k}(3)\right|+2}$. Since $2\left\lceil\left|\sigma_{2}^{k}(3)\right| / 2\right\rceil=\left|\sigma_{2}^{k}(3)\right|+1=\left\lfloor\left|\sigma_{2}^{k+1}(3)\right| / 2\right\rfloor$, we obtain inductively that $\psi_{\beta}^{\left|\sigma_{2}^{k}(3)\right|}((\infty, 0))$ starts with (1) for all $k \geq 0$.

For any $k \geq 0$, we have $a_{\left|\sigma_{2}^{k}(3)\right|} \geq 2, a_{\left|\sigma_{2}^{k}(3)\right|+1}=1, a_{\left|\sigma_{2}^{k}(3)\right|+2}=2$, and $t_{k}<0$. For any $k \geq 1$, this yields that

$$
\left.L_{\beta}\left(\left(|| \sigma_{2}^{k}(3) \mid / 2\right\rfloor,\left\lceil\left|\sigma_{2}^{k-1}(3)\right| / 2\right\rceil\right)\right)=t_{\left|\sigma_{2}^{k-1}(3)\right|}-t_{\left|\sigma_{2}^{k}(3)\right|-1}>-\frac{1}{\beta}+\frac{2}{\beta^{2}}+\frac{t_{0}}{\beta^{2}}+\frac{2}{\beta}-\frac{1}{\beta^{2}}>\frac{1}{\beta} .
$$

Let $k^{\prime}=-\left|\psi_{\beta}^{\left|\sigma_{2}^{k}(3)\right|}\right|$. Then we have $u_{k^{\prime}+j}=\left(\left\lfloor\left|\sigma_{2}^{k-j}(3)\right| / 2\right\rfloor,\left\lceil\left|\sigma_{2}^{k-j-1}(3)\right| / 2\right\rceil\right)$ for $0 \leq j<k$, thus $\left(y_{k^{\prime}}, y_{k^{\prime}+k}\right) \cap \mathbb{Z}_{-\beta}=\emptyset$ and $y_{k^{\prime}+k}-y_{k^{\prime}}>k / \beta$. Since $k \geq 1$ was chosen arbitrary, the distances between consecutive $(-\beta)$-integers are unbounded. 
Many other examples of sets $\mathbb{Z}_{-\beta}$ which are not relatively dense can be found by setting $a_{1} a_{2} \cdots=$ $\sigma_{3} \sigma_{2}^{\infty}(3)$ with a morphism $\sigma_{3}$ such that $\sigma_{3}(2)=2, \sigma_{3}(1)=1$, and $\sigma_{3}(3)$ is a suitable word of odd length.

We conclude the paper by stating the following three open problems, for which partial solutions are given in this section. Note that all the corresponding problems for positive bases have well-known, simple solutions, as mentioned above.

1. Characterise the sequences $a_{1} a_{2} \cdots$ which are possible $(-\beta)$-expansions of $\frac{-\beta}{\beta+1}$.

2. Characterise the numbers $\beta>1$ such that $\mathbb{Z}_{-\beta}$ is uniformly discrete.

3. Characterise the numbers $\beta>1$ such that $\mathbb{Z}_{-\beta}$ is relatively dense.

\section{References}

[1] P. Ambrož, D. Dombek, Z. Masáková \& E. Pelantová: Numbers with integer expansion in the numeration system with negative base. ArXiv:0912.4597v3 [math.NT].

[2] Č. Burdík, C. Frougny, J. P. Gazeau \& R. Krejcar (1998): Beta-integers as natural counting systems for quasicrystals. J. Phys. A 31(30), pp. 6449-6472, doi:10.1088/0305-4470/31/30/011.

[3] D. Dombek, Z. Masáková \& E. Pelantová: Number representation using generalized ( $-\beta)$-transformation. ArXiv:1102.3079v1 [cs.DM].

[4] P. Góra (2007): Invariant densities for generalized $\beta$-maps. Ergodic Theory Dynam. Systems 27(5), pp. 1583-1598, doi:10.1017/S0143385707000053.

[5] S. Ito \& T. Sadahiro (2009): Beta-expansions with negative bases. Integers 9, pp. A22, 239-259, doi:10. 1515/INTEG.2009.023.

[6] L. Liao \& W. Steiner: Dynamical properties of the negative beta-transformation. ArXiv:1101.2366v2 [math.DS].

[7] W. Parry (1960): On the $\beta$-expansions of real numbers. Acta Math. Acad. Sci. Hungar. 11, pp. 401-416, doi:10.1007/BF02020954.

[8] W. Steiner: On the structure of $(-\beta)$-integers. ArXiv:1011.1755v2 [math.NT]. 